\title{
Finite-Time Command-Filtered Approximation-Free Attitude Tracking Control of Rigid Spacecraft
}

\author{
Shuzong Xie $\cdot$ Qiang Chen $\cdot$ Xiongxiong He $\cdot$ Meiling Tao $\cdot$ Liang Tao
}

Received: date / Accepted: date

\begin{abstract}
This paper presents a finite-time commandfiltered approximation-free attitude tracking control for rigid spacecraft. A novel finite-time prescribed performance function (FTPPF) is first constructed to ensure that the attitude tracking errors converge to the predefined region in finite time. Then, a finite-time error compensation mechanism is constructed and incorporated into the backstepping control design, such that the differentiation of virtual control signals in recursive steps can be avoided to overcome the singularity issue. Compared with most of approximation-based attitude control methods, less computational burden and lower complexity are guaranteed by the proposed approximation-free control scheme due to the avoidance of using any function approximations. Simulation-
\end{abstract}

Shuzong Xie

College of Information Engineering, Zhejiang University of Technology

E-mail: xieshuzong@163.com

Qiang Chen

College of Information Engineering, Zhejiang University of Technology

E-mail: sdnjchq@zjut.edu.cn

Xiongxiong $\mathrm{He}$

College of Information Engineering, Zhejiang University of Technology

E-mail: hxx@zjut.edu.cn

Meiling Tao

Key Laboratory of Advanced Perception and Intelligent Control of High-end Equipment, Ministry of Education, Anhui Polytechnic University

E-mail: taomeilingsmile@163.com

Liang Tao

Key Laboratory of Advanced Perception and Intelligent Control of High-end Equipment, Ministry of Education, Anhui

Polytechnic University

E-mail: taoliang@ahpu.edu.cn $\mathrm{s}$ are given to illustrate the efficiency of the proposed method.

Keywords approximation-free control $\cdot$ attitude tracking $\cdot$ backstepping design $\cdot$ finite-time control . rigid spacecraft

\section{Introduction}

The attitude control of a rigid spacecraft is a significant and practical problem in various applications, such as formation flying, satellite surveillance, and earth observation [1]. A number of relevant works have been investigated for spacecraft attitude stabilization or tracking problems, including adaptive control [2], sliding mode control [3], robust control [4], event-triggered control [5], output feedback control [6], etc. All of the aforementioned methods are concerned with asymptotic stabilization or tracking of the spacecraft attitude, meaning that the attitudes will track the desired trajectories as time approaches the infinity.

Different from the aforementioned schemes, the finitetime control can guarantee the tracking error convergence within a finite time, and has been widely applied for the spacecraft systems (see [7-12], and references therein). In [10], a fast nonsingular terminal sliding mode control scheme was presented for the uncertain spacecraft, such that the convergence of attitude tracking errors could be achieved within finite time. In [11], an adaptive finite-time control with adding a power integrator was developed to guarantee that tracking errors could converge to the desired regions in finite time. In [12], an adaptive finite-time control law was presented by combining an integral disturbance observer and a terminal sliding mode surface, and the chattering problem could be eliminated. 
In most of the above-mentioned existing schemes, it was assumed that the models of rigid spacecraft were exactly or partially known. However, for practical spacecraft applications, system uncertainties and constraints have important influences on the safe operation and system performance. The existence of these factors makes it challenging to design an autonomous attitude controller, which is required to ensure a fast convergence rate of the tracking errors and satisfactory flight performance of the controlled spacecraft. To handle the uncertainties existing in the system dynamics, some function approximators including neural networks (NNs) and fuzzy logic systems (FLSs) are usually involved in the adaptive controllers design (see [1320], and references therein). In [19], a Chebyshev neural network-based finite-time control scheme was developed by constructing a terminal sliding mode surface, such that the uniform ultimate boundedness (UUB) of attitude tracking errors was guaranteed. In [20], a fast nonsingular terminal sliding mode control law was designed for rigid spacecraft, and the lumped system uncertainty including unknown inertia matrix and thrusters faults was approximated by the FLSs. Nevertheless, those approximation-based control approaches in literatures [13-20] may impose demanding computational burden due to their complicated structures in the implementation.

In addition, to achieve guaranteed transient tracking response, a constructive technique with a prescribed performance function (PPF) was presented in [21], where the transient tracking error can be rigorously maintained within a prescribed boundary. With the satisfactory characteristics, the PPF-based control has been applied to various nonlinear systems [22-26]. However, PPFs in the aforementioned literatures can only constrain the error convergence in exponential form, meaning that the system states can be maintained to the predefined region within infinite settling time. Recently, several finite-time prescribed performance functions (FTPPFs) have been presented to guarantee that the system states can be maintained to a predefined region in finite time $[27,28]$, but the approximation tools including FLSs and NNs may lead to the increase of computational burden. In [29], an approximation-free control (AFC) was developed for nonlinear systems without using any function NN or FLS approximations, and a proportional-like controller was designed with a simplified structure. Owing to this attractive property, the AFC method is easier to implement in various applications [30-35]. In [33], an adaptive error-constrained finite-time controller was presented for Lagrangian systems, where a piecewise function should be required in the controller design to handle the singularity problem, which would lead to a complex stability analysis. In [34], an approximation-free control scheme was proposed for rigid spacecraft, and the state variables can asymptotically converge to a small region around the origin. In [35], a model-free prescribed performance control approach was presented for flexible spacecraft to ensure the uniform ultimate boundedness (UUB) of attitude tracking errors, and the invertibility of model matrix should be always guaranteed.

Motivated by the above discussions, this paper proposes a novel finite-time approximation-free control ( $\mathrm{F}$ TAFC) scheme for the attitude tracking of rigid spacecraft. The main contributions are summarized as follows:

1) A novel finite-time prescribed performance function (FTPPF) is presented, such that the attitude tracking errors can be constrained to converge into the predefined region within finite time.

2) A finite-time approximation-free control (FTAFC) scheme is proposed by constructing a finite-time error compensation mechanism, and the singularity problem is directly avoided in the control design without using any piecewise functions.

3) Compared with the approximation-based control approaches in literatures [13-20], the unknown spacecraft dynamics can be accommodated effectively without employing any function approximations, and thus the proposed controller has less computational burden.

The remainder of the paper is structured as follows. Section II introduces the problem formulation and some preliminaries. The controller design and stability analysis are given in Section III. Comparative simulations are provided in Section IV, followed by some conclusions given in Section V.

Notations: Throughout this paper, $\|\cdot\|$ is the Euclidean norm of vectors or matrices. $I_{3} \in R^{3 \times 3}$ is a $3 \times 3$ identity matrix, and $\operatorname{diag}(\cdot)$ denotes the diagonal matrix. For a given vector $\nu=\left[\nu_{1}, \nu_{2}, \nu_{3}\right]^{T} \in R^{3}$ and a constant $\gamma \in R$, one defines $\operatorname{sig}^{\gamma}(\nu)=\left[\left|\nu_{1}\right|^{\gamma} \operatorname{sgn}\left(\nu_{1}\right),\left|\nu_{2}\right|^{\gamma}\right.$ $\left.\operatorname{sgn}\left(\nu_{2}\right),\left|\nu_{3}\right|^{\gamma} \operatorname{sgn}\left(\nu_{3}\right)\right]^{T}$ with $\operatorname{sgn}(\cdot)$ being the sign function. Besides, the matrix $\nu^{\times} \in R^{3 \times 3}$ is defined as $\nu^{\times}=$ $\left[0,-\nu_{3}, \nu_{2} ; \nu_{3}, 0,-\nu_{1} ;-\nu_{2}, \nu_{1}, 0\right]$.

\section{2 problem formulation and preliminaries}

\subsection{Attitude dynamics and kinematics of rigid spacecraft}

The attitude of a rigid spacecraft can be described by Modified Rodrigues Parameters(MRPs), which is for- 
mulated as $[36]$

$\sigma=n \tan \left(\frac{\epsilon(t)}{4}\right), \epsilon(t) \in(-2 \pi, 2 \pi)$

with $\sigma=\left[\sigma_{1}, \sigma_{2}, \sigma_{3}\right]^{T} \in R^{3}$ being the spacecraft attitude, $\epsilon(\cdot) \in R$ and $n=\left[n_{x}, n_{y}, n_{z}\right]^{T} \in R^{3}$ being the Euler angle and Euler axis, respectively. The MRPs vector $\sigma$ can be mapped to its shadow counterpart $\sigma^{s}$ with the following relationship [37]

$\sigma^{s}=\frac{1}{\sigma^{T} \sigma} \sigma$

and $\sigma$ is guaranteed to be bounded within a unit sphere through switching the MRPs to $\sigma^{s}$ in (2) as $\sigma^{T} \sigma>1$, such that the global singularity-free rotation representation is ensured. In terms of the MRPs (1), the spacecraft attitude kinematic and dynamic equations are given by

$\dot{\sigma}=G(\sigma) \omega$

$J \dot{\omega}=-\omega^{\times} J \omega+u+d$

where $G(\sigma) \in R^{3 \times 3}$ is a Jacobian matrix defined by $G(\sigma)=\frac{1}{2}\left[\frac{1-\sigma^{T} \sigma}{2} I_{3}+\sigma^{\times}+\sigma \sigma^{T}\right], \omega=\left[\omega_{1}, \omega_{2}, \omega_{3}\right]^{T} \in R^{3}$ represents the angular velocity; $J \in R^{3 \times 3}$ denotes a symmetric, positive and bounded inertia matrix; $u=$ $\left[u_{1}, u_{2}, u_{3}\right]^{T} \in R^{3}$ is the control torque, and $d=\left[d_{1}, d_{2}\right.$, $\left.d_{3}\right]^{T} \in R^{3}$ represents unknown but bounded disturbances.

\subsection{Relative attitude error dynamics and kinematics}

Define $\sigma_{d}=\left[\sigma_{d 1}, \sigma_{d 2}, \sigma_{d 3}\right]^{T} \in R^{3}$ as the MRPs of the desired attitude, and $\omega_{d}=\left[\omega_{d 1}, \omega_{d 2}, \omega_{d 3}\right]^{T} \in R^{3}$ as a time-varying and continuous desired angular velocity. In this paper, we assume that $\omega_{d}$ and its first-order differentiation are both continuous and bounded.

The attitude tracking error and angular velocity error represented by MRPs are defined as $e=\left[e_{1}, e_{2}, e_{3}\right]^{T} \in$ $R^{3}$ and $\omega_{e}=\left[\omega_{e 1}, \omega_{e 2}, \omega_{e 3}\right]^{T} \in R^{3}$, respectively, which can be formulated as [37]

$e=\sigma \otimes \sigma_{d}^{-1}=\frac{\sigma_{d}\left(\sigma^{T} \sigma-1\right)+\sigma\left(1-\sigma_{d}^{T} \sigma_{d}\right)-2 \sigma_{d}^{\times}}{1+\sigma_{d}^{T} \sigma_{d} \sigma^{T} \sigma+2 \sigma_{d}^{T} \sigma}$

$\omega_{e}=\omega-R_{d}^{b} \omega_{d}$

where $R_{d}^{b}=I_{3}-4\left(\left(1-e^{T} e\right) /\left(1+e^{T} e\right)^{2}\right) e^{\times}+8\left(e^{\times}\right)^{2} /(1+$ $\left.e^{T} e\right)^{2}$ is the corresponding direction cosine matrix.

From (3)-(6), the dynamics and kinematics of the relative attitude error are formulated as

$\dot{e}=G(e) \omega_{e}$
$J \dot{\omega}_{e}=-\omega^{\times} J \omega-J R_{d}^{b} \dot{\omega}_{d}+J \omega_{e}^{\times} R_{d}^{b} \omega_{d}+u+d$

Then, Eq.(8) can be rewritten as

$\dot{\omega}_{e}=-J^{-1} \omega^{\times} J \omega-R_{d}^{b} \dot{\omega}_{d}+\omega_{e}^{\times} R_{d}^{b} \omega_{d}+J^{-1} u+J^{-1} d$

The time derivative of $\dot{e}$ along (7) yields

$$
\begin{aligned}
\ddot{e}= & \dot{G}(e) \omega_{e}+G(e) \dot{\omega}_{e} \\
= & \dot{G}(e) \omega_{e}+G(e)\left[-J^{-1} \omega^{\times} J \omega-R_{d}^{b} \dot{\omega}_{d}+\omega_{e}^{\times} R_{d}^{b} \omega_{d}\right. \\
& \left.+J^{-1} u+J^{-1} d\right]
\end{aligned}
$$

Define $x_{1}=e, x_{2}=\dot{e}$, and then Eq.(10) can be rewritten as

$\left\{\begin{array}{l}\dot{x}_{1}=x_{2} \\ \dot{x}_{2}=\Phi\left(x_{1}, x_{2}, \omega_{d}, \dot{\omega}_{d}\right)+G\left(x_{1}\right) J^{-1} d+G\left(x_{1}\right) J^{-1} u\end{array}\right.$

where $\Phi\left(x_{1}, x_{2}, \omega_{d}, \dot{\omega}_{d}\right)=\dot{G}\left(x_{1}\right) \omega_{e}+G\left(x_{1}\right)\left[-J^{-1} \omega^{\times} J \omega-\right.$ $\left.R_{d}^{b} \dot{\omega}_{d}+\omega_{e}^{\times} R_{d}^{b} \omega_{d}\right]$, and $\dot{G}\left(x_{1}\right)=\frac{1}{2}\left[-x_{1}^{T} \dot{x}_{1} I_{3}+\dot{x}_{1}^{\times}+\right.$ $\left.\dot{x}_{1} x_{1}^{T}+x_{1} \dot{x}_{1}^{T}\right]$ is bounded by

$$
\begin{aligned}
\left\|\dot{G}\left(x_{1}\right)\right\|= & \frac{1}{2}\left\|-x_{1}^{T} \dot{x}_{1} I_{3}+\dot{x}_{1}^{\times}+\dot{x}_{1} x_{1}^{T}+x_{1} \dot{x}_{1}^{T}\right\| \\
\leq & \frac{1}{2}\left[\left\|x_{1}^{T} x_{2} I_{3}\right\|+\left\|x_{2}^{\times}\right\|+\left\|x_{2} x_{1}^{T}\right\|+\left\|x_{1} x_{2}^{T}\right\|\right] \\
\leq & \frac{1}{2}\left[\left\|x_{1}\right\| \cdot\left\|x_{2}\right\|+\left\|x_{2}\right\|+\left\|x_{2}\right\| \cdot\left\|x_{1}\right\|\right. \\
& \left.+\left\|x_{1}\right\| \cdot\left\|x_{2}\right\|\right] \\
= & \frac{1}{2}\left[\left\|x_{2}\right\|+3\left\|x_{1}\right\| \cdot\left\|x_{2}\right\|\right]
\end{aligned}
$$

The Jacobin matrix $G\left(x_{1}\right)$ has the following properties[38]:

$G\left(x_{1}\right)^{-1}=\frac{16}{\left(1+x_{1}^{T} x_{1}\right)^{2}} G\left(x_{1}\right)^{T}$

$G\left(x_{1}\right)^{T} G\left(x_{1}\right)=\left(\frac{1+x_{1}^{T} x_{1}}{4}\right)^{2} I_{3}$.

Remark 1: There exist some different attitude representations for spacecraft, including quaternions and MRPs, etc [39]. Although the quaternion based method has been widely used to present the spacecraft attitude, the four elements are not independent due to the existence of constraints, which leading to the redundancy in the attitude calculation. Compared to quaternions, the MRPs formulation is more computationally efficient in the real-time attitude calculation, such that the MRPs is adopted to describe the spacecraft attitude system (3) and (4) in this paper. 


\subsection{Preliminaries}

Consider the initial value problem [40] as

$\dot{\zeta}(t)=h(t, \zeta(t)), \zeta(0)=\zeta^{0} \in \Omega_{\zeta}$

with $h: \Re_{+} \times \Omega_{\zeta} \rightarrow \Re^{n}$ being a continuous function vector, and $\Omega_{\zeta} \in \Re^{n}$ being a nonempty open set.

Definition 1 [40]: The solution of the initial value problem (15) is maximal if it has no proper right extension that is also a solution of (15).

Lemma 1 [40]: If the function vector $h(t, \zeta(t))$ satisfies the following conditions:

- locally Lipschitz on $\zeta(t)$;

- continuous on time for each fixed $\zeta \in \Omega_{\zeta}$; and

- locally integrable on time for each fixed $\zeta \in \Omega_{\zeta}$.

Then, there exists a maximal solution $\zeta(t):\left[0, \tau_{\max }\right) \rightarrow$ $\Omega_{\zeta}$ on the time interval $\left[0, \tau_{\max }\right)$ with $\tau_{\max }>0$ and $\zeta(t) \in \Omega_{\zeta}, \forall t \in\left[0, \tau_{\max }\right)$.

Proposition 1 [40]: When the conditions of Lemma 1 hold, for a maximal solution $\zeta(t):\left[0, \tau_{\max }\right) \rightarrow \Omega_{\zeta}$ on the time interval $\left[0, \tau_{\max }\right)$ with $\tau_{\max }>0$ and for any compact set $\bar{\Omega}_{\zeta} \subset \Omega_{\zeta}$, there exists a time instant $t_{1} \in\left[0, \tau_{\max }\right)$ satisfying $\zeta\left(t_{1}\right) \notin \bar{\Omega}_{\zeta}$.

\subsection{Finite-Time Command Filter}

To construct a finite-time error compensation mechanism, the following command filter is employed in the controller design [41]

$$
\begin{aligned}
\dot{x}_{c} & =v \\
v & =-\beta_{1}\left|x_{c}-\alpha\right|^{1 / 2} \operatorname{sgn}\left(x_{c}-\alpha\right)+L \\
\dot{L} & =-\beta_{2} \operatorname{sgn}(\varphi-v)
\end{aligned}
$$

where $\alpha$ is the input of the finite-time command filter, and $x_{c}$ and $v$ are the outputs; $\beta_{1}$ and $\beta_{2}$ are positive constants.

As proved in [41], by properly choosing $\beta_{1}$ and $\beta_{2}$, the following equalities hold within a finite time

$x_{c}=\alpha_{0}, v=\dot{\alpha}_{0}$

where $\alpha_{0}$ is the ideal input without noise (i.e., $\alpha_{0}=\alpha$ ). In practice, the measured input is usually affected by noise such that $\alpha \neq \alpha_{0}$ holds, and then the following result is obtained.

Lemma 2[41]: For the finite-time command filter given in (16), when there is bounded measurement noise such that $\left|\alpha-\alpha_{0}\right| \leq \kappa_{1}$, then the finite-time convergence of the following inequalities hold:

$$
\begin{aligned}
& \left|x_{c}-\alpha_{0}\right| \leq \varsigma_{1} \kappa_{1}=\varpi_{1} \\
& \left|v-\dot{\alpha}_{0}\right| \leq \varsigma_{2} \kappa_{1}^{1 / 2}=\varpi_{2}
\end{aligned}
$$

where $\kappa_{1}, \varsigma_{1}, \varsigma_{2}, \varpi_{1}$ and $\varpi_{2}$ are positive constants.

\section{Finite-Time Approximation-Free Control}

In this section, a finite-time approximation-free control (FTAFC) is developed for system (11) to maintain the transient tracking performance as well as steadystate tracking performance, where the finite-time stability analysis is also provided.

\subsection{Finite-Time Prescribed Performance Function}

To maintain the attitude tracking errors of the spacecraft system within a predefined time and boundary, an essential definition is defined first.

Definition 2: If a smooth function $\rho(t)$ satisfies the following conditions:

$-\rho(t)>0, \dot{\rho}(t)<0$ for $t \in\left[0, T_{0}\right]$

- $\lim _{t \rightarrow T_{0}} \rho(t)=\rho_{T_{0}}$ with $\rho_{T_{0}}$ being an arbitrarily small positive constant; and

- $\rho(t)=\rho_{T_{0}}$ for $\forall t \geq T_{0}$ with $T_{0}$ being the settling time.

Then, this function is called finite-time prescribed performance function (FTPPF).

According to Definition 2, a novel FTPPF $\rho(t)$ in this paper is constructed as

$\rho(t)= \begin{cases}(a t-b)^{2 c}+\rho_{T_{0}}, & 0 \leq t<T_{0} \\ \rho_{T_{0}}, & t \geq T_{0}\end{cases}$

where $c$ is a positive integer, $a=\left(\rho_{0}-\rho_{T_{0}}\right)^{\frac{1}{2 c}} / T_{0}$ and $b=\left(\rho_{0}-\rho_{T_{0}}\right)^{\frac{1}{2 c}}$ are calculated by the following constraint relationships:

$\left\{\begin{array}{l}\rho(0)=\rho_{0}, \\ \rho\left(t \mid t \geq T_{0}\right)=\rho_{T_{0}}, \dot{\rho}\left(t \mid t \geq T_{0}\right)=0 .\end{array}\right.$

where $\rho(0)=\rho_{0}=(-b)^{2 c}+\rho_{T_{0}}$ is initial value of FTPPF.

Remark 2: In the literature, the following form of $\mathrm{PPF}$ is commonly used [30-35]:

$\rho(t)=\left(\rho_{0}-\rho_{\infty}\right) e^{-a t}+\rho_{\infty}$

where $\rho_{\infty}>\rho_{0}>0, \rho_{0}=\rho(0)$, and $a$ is a positive constant. Note that (21) is an exponential form, which means that the settling time of PPF is infinite and the convergence rate of $\mathrm{PPF}$ is slower.

The corresponding comparison of different prescribed performance functions is shown in Fig.1. It can be seen from Fig. 1 that the settling time of FTPPF is fixed, while the settling time of PPF is infinite. Then, the prescribed bound of the attitude tracking error $e_{i}$ with 


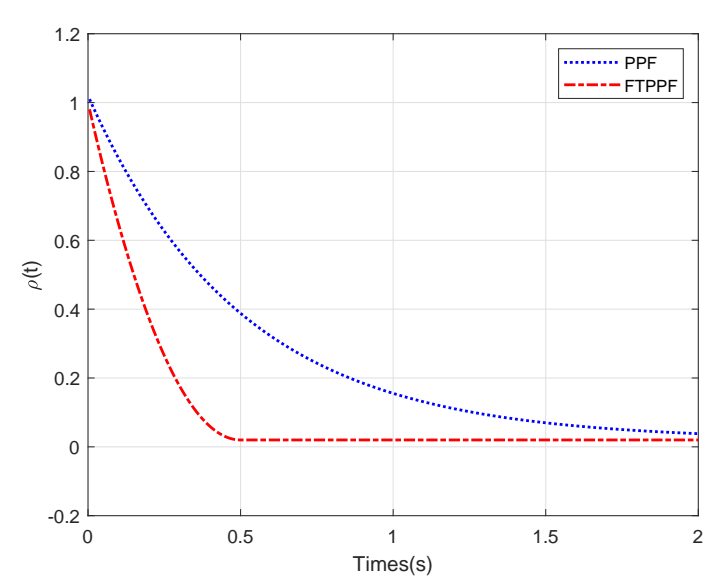

Fig. 1 Comparison of different prescribed performance functions

$i=1,2,3$ is retained by the following constrained condition:

$-\underline{\delta} \rho(t)<e_{i}(t)<\bar{\delta} \rho(t), \forall t>0$

where $\bar{\delta}$ and $\underline{\delta}$ are positive constants denoting the upper and lower bounds of overshoot, respectively.

To retain the predefined response (22), a strictly monotonically increasing and smooth function $S\left(\zeta_{i}\right)$ is chosen to satisfy the following conditions:

$$
\left\{\begin{array}{l}
-\underline{\delta}<S\left(\zeta_{i}\right)<\bar{\delta}, \forall \zeta_{i} \in R \\
\lim _{t \rightarrow+\infty} S\left(\zeta_{i}\right)=\bar{\delta}, \text { and } \lim _{t \rightarrow-\infty} S\left(\zeta_{i}\right)=-\underline{\delta} .
\end{array}\right.
$$

where $\zeta_{i}=\frac{e_{i}(t)}{\rho(t)}$ is a normalized error.

Then, the control problem with the error boundary condition (22) is transformed into an equivalent "unconstrained" control problem of the transformed error $\zeta_{i}(t)$. According to (23), the transformation function $S\left(\zeta_{i}\right)$ can be chosen as

$S\left(\zeta_{i}\right)=\frac{\bar{\delta} \exp \left(\zeta_{i}\right)-\underline{\delta} \exp \left(-\zeta_{i}\right)}{\exp \left(\zeta_{i}\right)+\exp \left(-\zeta_{i}\right)}$

and thus the inverse function of (24) is given by

$\varepsilon_{i}=S^{-1}\left(\zeta_{i}\right)=\frac{1}{2} \ln \left(\frac{\delta}{\bar{\delta}-\zeta_{i}}\right)$

Remark 2: Compared with the PPF-based methods, the presented FTPPF (19) has the following two merits: 1) FTPPF (19) can converge to the predefined region in finite time; 2) The settling time can be specified by the designer, which is more efficient for practical applications.

Remark 3: According to (19), the FTPPF parameters include $\rho_{0}, T_{0}, \rho_{T_{0}}$ and $c$. Generally, $\rho_{0}=\rho(0)=$ $(-b)^{2 c}+\rho_{T_{0}}$ is the initial condition, and it should be selected to satisfy the condition $\rho_{0}>\left|e_{i}(0)\right| \cdot \rho_{T_{0}}>0$ is the maximum allowable size of ultimate error, which can be set arbitrarily small to achieve satisfactory tracking precision; $T_{0}>0$ is the settling time from initial error to the ultimate error, which can be set relatively large initially and then reduced gradually. $c$ defines the decreasing rate of $\rho(t)$ during the initial phase, which can be increased from $c=1$. Thus, by adjusting the FTPPF parameters $\rho_{0}, T_{0}, \rho_{T_{0}}$ and $c$ properly, the finite-time prescribed performance can be ensured.

\subsection{Controller Design}

To ensure the spacecraft finite-time transient tracking performance as well as steady-state tracking performance, the improved error transformation function (25) is incorporated into the control design. The detailed design steps are given as follows.

Step 1: Define virtual state $z_{1}$ as

$z_{1}=x_{1}$

where $z_{1}=\left[z_{11}, z_{12}, z_{13}\right]^{T}$.

The time derivative of $z_{1}$ along (26) is

$\dot{z}_{1}=x_{2}$

Define the first normalized error as $\zeta_{1 i}=\frac{z_{1 i}}{\rho_{1}}$, and then from (24) and (25), the transformed error signal is obtained as

$\varepsilon_{1 i}=\frac{1}{2} \ln \left(\frac{\underline{\delta}+\zeta_{1 i}}{\bar{\delta}-\zeta_{1 i}}\right), i=1,2,3$

where $\underline{\delta}$ and $\bar{\delta}$ are positive constants, and $\rho_{1}(t)$ is a FTPPF given by (19), whose parameter $z_{1 i}$ is set to fulfill the initial condition $-\underline{\delta} \rho_{1}(0)<z_{1 i}(0)<\bar{\delta} \rho_{1}(0)$.

Differentiating $\varepsilon_{1 i}$ along (28) leads to

$$
\begin{aligned}
\dot{\varepsilon}_{1 i} & =\frac{1}{2} \frac{\dot{\zeta}_{1 i}\left(\bar{\delta}-\zeta_{1 i}\right)-\left(\underline{\delta}+\zeta_{1 i}\right)\left(-\dot{\zeta}_{1 i}\right)}{\left(\bar{\delta}-\zeta_{1 i}\right)^{2}} \cdot \frac{\bar{\delta}-\zeta_{1 i}}{\underline{\delta}+\zeta_{1 i}} \\
& =\frac{(\bar{\delta}+\underline{\delta}) \dot{\zeta}_{1 i}}{2\left(\bar{\delta}-\zeta_{1 i}\right)\left(\underline{\delta}+\zeta_{1 i}\right)} \\
& =g_{1 i}\left(x_{2 i}-\zeta_{1 i} \dot{\rho}_{1}\right)
\end{aligned}
$$

where $g_{1 i}=\frac{(\bar{\delta}+\underline{\delta})}{2 \rho_{1}\left(\bar{\delta}-\zeta_{1 i}\right)\left(\underline{\delta}+\zeta_{1 i}\right)}$ with $i=1,2,3$.

Then, Eq.(29) can be rewritten as

$\dot{\varepsilon}_{1}=g_{1}\left(x_{2}-\zeta_{1} \dot{\rho}_{1}\right)$

where $\dot{\varepsilon}_{1}=\left[\dot{\varepsilon}_{11}, \dot{\varepsilon}_{12}, \dot{\varepsilon}_{13}\right]^{T}$ and $g_{1}=\operatorname{diag}\left(g_{11}, g_{12}, g_{13}\right)$.

Define the intermediate error $z_{2}$ as

$$
z_{2}=x_{2}-x_{c}
$$


where $z_{2}=\left[z_{21}, z_{22}, z_{23}\right]^{T}$, and $x_{c}$ is the output of finitetime command filter, which is designed as

$$
\begin{aligned}
\dot{x}_{c i} & =v_{i} \\
\dot{v}_{i} & =-\beta_{1}\left|x_{c i}-\alpha_{i}\right|^{1 / 2} \operatorname{sgn}\left(x_{c i}-\alpha_{i}\right)+L_{i} \\
\dot{L}_{i} & =-\beta_{2} \operatorname{sgn}\left(L_{i}-\dot{v}_{i}\right), \quad i=1,2,3
\end{aligned}
$$

where $\beta_{1}$ and $\beta_{2}$ are positive constants, $x_{c i}$ and $v_{i}$ respectively denote the finite-time command filter's outputs for estimating the virtual control law $\alpha_{i}$ and its derivative $\dot{\alpha}_{i}$ to be designed below.

To retain the boundedness and finite-time convergence of $\varepsilon_{1 i}$, the virtual control law $\alpha=\left[\alpha_{1}, \alpha_{2}, \alpha_{3}\right]^{T}$ is designed as

$\alpha=-k_{1} \varepsilon_{1}-\tau_{1} \operatorname{sig}^{\gamma}\left(s_{1}\right)$

where $\varepsilon_{1}=\left[\varepsilon_{11}, \varepsilon_{12}, \varepsilon_{13}\right]^{T}, k_{1}$ and $\tau_{1}$ are positive constants, $0<\gamma<1$, and $s_{1}=\left[s_{11}, s_{12}, s_{13}\right]^{T}$ is the compensated tracking error defined as

$s_{1}=\varepsilon_{1}-\xi_{1}$

where $\xi_{1}=\left[\xi_{11}, \xi_{12}, \xi_{13}\right]^{T}$ is the error compensation signal given by

$\dot{\xi}_{1}=g_{1}\left[-k_{1} \xi_{1}-l_{1} \operatorname{sgn}\left(\xi_{1}\right)+x_{c}-\alpha\right]$

where $l_{1}$ is a positive constant.

Substituting (31), (33) and (35) into (30) yields

$$
\begin{aligned}
\dot{\varepsilon}_{1} & =g_{1}\left[x_{2}-\zeta_{1} \dot{\rho}_{1}\right] \\
& =g_{1}\left[x_{c}+z_{2}-\zeta_{1} \dot{\rho}_{1}\right] \\
& =g_{1}\left[x_{c}-\alpha+\alpha+z_{2}-\zeta_{1} \dot{\rho}_{1}\right] \\
& =g_{1}\left[-k_{1} \varepsilon_{1}-\tau_{1} \operatorname{sig}^{\gamma}\left(s_{1}\right)+\left(x_{c}-\alpha\right)+z_{2}-\zeta_{1} \dot{\rho}_{1}\right]
\end{aligned}
$$

Step 2: The time derivative of $z_{2}$ along (31) is

$\dot{z}_{2}=\dot{x}_{2}-\dot{x}_{c}$

Substituting (11) into (37) results in

$\dot{z}_{2}=\Phi\left(x_{1}, x_{2}, \omega_{d}, \dot{\omega}_{d}\right)+G\left(x_{1}\right) J^{-1} d+G\left(x_{1}\right) J^{-1} u-\dot{x}_{c}$

Define the second normalized error as $\zeta_{2 i}=\frac{z_{2 i}}{\rho_{2}}$, and the corresponding transformed error signal is given by

$\varepsilon_{2 i}=\frac{1}{2} \ln \left(\frac{\underline{\delta}+\zeta_{2 i}}{\bar{\delta}-\zeta_{2 i}}\right), i=1,2,3$

where $\rho_{2}(t)$ is a FTPPF given by (19), whose parameter $z_{2 i}$ is set to satisfy the initial condition $-\underline{\delta} \rho_{2}(0)<$ $z_{2 i}(0)<\bar{\delta} \rho_{2}(0), i=1,2,3$.
Differentiating $\varepsilon_{2 i}$ along (39) yields

$$
\begin{aligned}
\dot{\varepsilon}_{2 i} & =\frac{1}{2} \frac{\dot{\zeta}_{2 i}\left(\bar{\delta}-\zeta_{2 i}\right)-\left(\underline{\delta}+\zeta_{2 i}\right)\left(-\dot{\zeta}_{2 i}\right)}{\left(\bar{\delta}-\zeta_{2 i}\right)^{2}} \cdot \frac{\bar{\delta}-\zeta_{2 i}}{\underline{\delta}+\zeta_{2 i}} \\
& =\frac{(\bar{\delta}+\underline{\delta}) \dot{\zeta}_{2 i}}{2\left(\bar{\delta}-\zeta_{2 i}\right)\left(\underline{\delta}+\zeta_{2 i}\right)} \\
& =g_{2 i}\left(\dot{z}_{2 i}-\zeta_{2 i} \dot{\rho}_{2}\right)
\end{aligned}
$$

where $g_{2 i}=\frac{(\bar{\delta}+\underline{\delta})}{2 \rho_{2}\left(\bar{\delta}-\zeta_{2 i}\right)\left(\underline{\delta}+\zeta_{2 i}\right)}$ with $i=1,2,3$.

Then, Eq.(40) can be rewritten as

$\dot{\varepsilon}_{2}=g_{2}\left(\dot{z}_{2}-\zeta_{2} \dot{\rho}_{2}\right)$

where $\dot{\varepsilon}_{2}=\left[\dot{\varepsilon}_{21}, \dot{\varepsilon}_{22}, \dot{\varepsilon}_{23}\right]^{T}$ and $g_{2}=\operatorname{diag}\left(g_{21}, g_{22}, g_{23}\right)$.

Substituting (38) into (41) yields

$$
\begin{aligned}
\dot{\varepsilon}_{2}= & g_{2}\left[\Phi\left(x_{1}, x_{2}, \omega_{d}, \dot{\omega}_{d}\right)+G\left(x_{1}\right) J^{-1} d+G\left(x_{1}\right) J^{-1} u\right. \\
& \left.-\dot{x}_{c}-\zeta_{2} \dot{\rho}_{2}\right]
\end{aligned}
$$

Similar to the developments given in Step 1, the actual control law $u$ is designed as

$u=J G\left(x_{1}\right)^{-1}\left[-k_{2} \varepsilon_{2}-\tau_{2} \operatorname{sig}^{\gamma}\left(\varepsilon_{2}\right)\right]$

where $\varepsilon_{2}=\left[\varepsilon_{21}, \varepsilon_{22}, \varepsilon_{23}\right]^{T}, k_{2}$ and $\tau_{2}$ are positive constants.

Substituting (43) into (42) results in

$$
\begin{aligned}
\dot{\varepsilon}_{2}= & g_{2}\left[\Phi\left(x_{1}, x_{2}, \omega_{d}, \dot{\omega}_{d}\right)+G\left(x_{1}\right) J^{-1} d-k_{2} \varepsilon_{2}-\right. \\
& \left.\tau_{2} \operatorname{sig}^{\gamma}\left(\varepsilon_{2}\right)-\dot{x}_{c}-\zeta_{2} \dot{\rho}_{2}\right]
\end{aligned}
$$

To show the boundedness of $\zeta_{1}, \zeta_{2}$ and the convergence of $z_{1}$ and $z_{2}$, the time derivatives of the normalized errors $\zeta_{1}, \zeta_{2}$ are calculated as

$$
\begin{aligned}
\dot{\zeta}_{1} & =\frac{d\left(z_{1} / \rho_{1}\right)}{d t}=\frac{1}{\rho_{1}}\left(\dot{z}_{1}-\zeta_{1} \dot{\rho}_{1}\right) \\
& =\frac{1}{\rho_{1}}\left(\zeta_{2} \rho_{2}+x_{c}-\zeta_{1} \dot{\rho}_{1}\right) \\
& =h_{1}\left(t, \zeta_{1}, \zeta_{2}\right)
\end{aligned}
$$

and

$$
\begin{aligned}
\dot{\zeta}_{2} & =\frac{d\left(z_{2} / \rho_{2}\right)}{d t}=\frac{1}{\rho_{2}}\left(\dot{z}_{2}-\zeta_{2} \dot{\rho}_{2}\right) \\
& =\frac{1}{\rho_{2}}\left[\ddot{x}_{1}-\dot{x}_{c}-\zeta_{2} \dot{\rho}_{2}\right] \\
& =h_{2}\left(t, \zeta_{1}, \zeta_{2}\right)
\end{aligned}
$$

Define an error vector $\zeta=\left[\zeta_{1}^{T}, \zeta_{2}^{T}\right]^{T}$, one has

$\dot{\zeta}(t)=h(t, \zeta)=\left[\begin{array}{l}h_{1}\left(t, \zeta_{1}, \zeta_{2}\right) \\ h_{2}\left(t, \zeta_{1}, \zeta_{2}\right)\end{array}\right]$

It is worth noting that the initial FTPPFs $\rho_{1}(t)$ and $\rho_{2}(t)$ should be chosen to satisfy the conditions 
$\left|z_{1 i}\right|<\min \{\underline{\delta}, \bar{\delta}\} \rho_{1}(0)$ and $\left|z_{2 i}\right|<\min \{\underline{\delta}, \bar{\delta}\} \rho_{2}(0), i=$ $1,2,3$. Hence, we can define an open and nonempty set $\Omega_{\zeta}=(-\underline{\delta}, \bar{\delta})_{1} \times \ldots \times(-\underline{\delta}, \bar{\delta})_{6}$, such that $\zeta(0) \in \Omega_{\zeta}$ is true. Moreover, due to the continuity and differentiability of system dynamics with respect to the states and FTPPFs $\rho_{1}(t)$ and $\rho_{2}(t), h(t, \zeta)$ is bounded and continuously differentiable on $t$ and locally Lipschitz on $\zeta$ over the set $\Omega_{\zeta}$. According to Lemma 1, a maximal solution $\zeta(t)$ of $(47)$ exists on the time interval $\left[0, \tau_{\max }\right)$, such that $\zeta$ is bounded by $\left|\zeta_{1 i}\right|<\min \{\underline{\delta}, \bar{\delta}\}$ and $\left|\zeta_{2 i}\right|<\min \{\underline{\delta}, \bar{\delta}\}$ for $\forall t \in\left[0, \tau_{\max }\right)$ with $i=1,2,3$. Consequently, $g_{1 i}$ and $g_{2 i}$ are bounded, there exist four positive constants $g_{m 1}, g_{M 1}, g_{m 2}$ and $g_{M 2}$ satisfying $0<$ $g_{m 1}<g_{1 i}<g_{M 1}$ and $0<g_{m 2}<g_{2 i}<g_{M 2}$ with $i=1,2,3$.

Remark 4: It is well-known that function approximators including FLS and RBFNNs are widely employed to approximate the nonlinear dynamic in the controlled systems, but there are not general guidelines for the parameter tuning of FLS and RBFNN weights. Besides, the design of the adaptation laws also takes a long time to ensure the convergence, leading to potentially sluggish transient responses [42]. From (33) and (43), it is seen that the proposed control structure has a simple cascade proportional-like structure without using any function approximations, such that the computational costs in the control implementation can be reduced.

Remark 5: Different from the existing classical AFC schemes [29-35], in which the error convergence is guaranteed when the time goes to infinity, the controller (43) can ensure the finite-time convergence of the attitude tracking errors without triggering any potential singularity issues. In addition, the design of the error compensation mechanism (35) is to compensate for the influence of the filtering error $x_{c i}-\alpha_{i}, i=1,2,3$ caused by the finite-time command filter (16).

\subsection{Stability Analysis}

To analyze the finite-time stability of the closed-loop control system, some essential lemmas are first provided as follows.

Lemma 3[10]: Given a system $\dot{x}=f(x)$, if there exist a continuous function $V(x)$ and scalars $\varrho_{1}>0, \varrho_{2}>$ $0,0<\gamma<1$ and $0<\eta<\infty$ satisfying $\dot{V}(x) \leq-\varrho_{1} V-$ $\varrho_{2} V^{\gamma}+\eta$, then the trajectory of the system $\dot{x}=f(x)$ is practical finite-time stable, residual set of the solution is calculated as $\left\{\lim _{t \rightarrow T} x \mid V(x) \leq \min \left\{\left(\frac{\eta}{(1-\kappa) \varrho_{1}}\right),\left(\frac{\eta}{(1-\kappa) \varrho_{2}}\right)^{\frac{1}{\gamma}}\right\}\right\}$ where $\kappa$ satisfies $0<\kappa<1$. The settling time is bound- ed by

$$
\begin{gathered}
T \leq \max \left\{t_{0}+\frac{1}{\kappa \varrho_{1}(1-\gamma)} \ln \frac{\kappa \varrho_{1} V^{1-\gamma}\left(t_{0}\right)+\varrho_{2}}{\varrho_{2}},\right. \\
\left.t_{0}+\frac{1}{\varrho_{1}(1-\gamma)} \ln \frac{\kappa \varrho_{1} V^{1-\gamma}\left(t_{0}\right)+\kappa \varrho_{2}}{\kappa \varrho_{2}}\right\} .
\end{gathered}
$$

Lemma 4 [37]: For all positive numbers $x_{i}, i=1,2, \ldots, n$ and there exists a constant $0<p<1$, such that the following inequality holds

$\left(\left|x_{1}\right|+\ldots+\left|x_{n}\right|\right)^{p}<\left|x_{1}\right|^{p}+\ldots+\left|x_{n}\right|^{p}$.

The following two theorems are proved to show the main results of this paper.

Theorem 1: Consider the error compensation mechanisms (35), the signals $\xi_{1}$ can converge to origin within the finite time.

Proof: Choose a Lyapunov function candidate as

$V_{1}=\frac{1}{2} \xi_{1}^{T} \xi_{1}$

Differentiating (49) yields

$\dot{V}_{1}=\xi_{1}^{T} \dot{\xi}_{1}$

Substituting (35) into (50) and according to the Lemma 2 result in

$$
\begin{aligned}
\dot{V}_{1} & =\xi_{1}^{T} g_{1}\left[-k_{1} \xi_{1}-l_{1} \operatorname{sgn}\left(\xi_{1}\right)+x_{c}-\alpha\right] \\
& \leq \sum_{i=1}^{3} g_{1 i}\left[-k_{1} \xi_{1 i}^{2}-\left(l_{1}-\varpi\right)\left|\xi_{1 i}\right|\right] \\
& \leq \varrho_{1} V_{1}-\varrho_{2} V_{1}^{\frac{1}{2}}
\end{aligned}
$$

where $\varrho_{1}=2 k_{1} g_{1 m}$ is a positive constant, $\varrho_{2}=\sqrt{2}\left(l_{1}-\right.$ $\varpi) g_{m 1}>0$ holds when choosing appropriate constant $l_{1}$ to satisfy $l_{1}-\varpi>0$. According to Lemma 3 , it is concluded that $\xi_{1 i}$ can converge to origin with the following finite settling time

$T_{1} \leq \frac{2}{\varrho_{1}} \ln \left(1+\frac{\varrho_{1}}{\varrho_{2}}\left[V_{1}(0)\right]^{\frac{1}{2}}\right)$.

This completes the proof.

Theorem 2: Considering rigid spacecraft system (11), we construct the virtual controller (33), actual controller (43) and error compensation mechanism (35). If the initial conditions $z_{1 i}(0)<\min \{\underline{\delta}, \bar{\delta}\} \rho_{1}(0)$ and $z_{2 i}(0)<\min \{\underline{\delta}, \bar{\delta}\} \rho_{2}(0)$ are satisfied, then all signals in the closed-loop system are bounded, and the attitude tracking error $e_{i}$ is maintained within the prescribed region (22) and converge into the sufficiently small region around origin in finite time.

\}, Proof: The whole proof process contains four steps. In Step 1-Step 3, all signals in the closed-loop system 
are proved to be bounded, and the proof of the finitetime convergence of the attitude tracking error $e_{i}$ is given in Step 4.

Step 1: Construct a Lyapunov function candidate as

$V_{2}=\frac{1}{2} s_{1}^{T} s_{1}$

Differentiating (53) yields

$$
\begin{aligned}
\dot{V}_{2} & =s_{1}^{T} \dot{s}_{1} \\
& =s_{1}^{T}\left(\dot{\varepsilon}_{1}-\dot{\xi}_{1}\right)
\end{aligned}
$$

Substituting (35) and (36) into (54) yields

$$
\begin{aligned}
\dot{V}_{2} & =s_{1}^{T} g_{1}\left[z_{2}-k_{1} s_{1}-\tau_{1} \operatorname{sig}^{\gamma}\left(s_{1}\right)-\zeta_{1} \dot{\rho}_{1}+l_{1} \operatorname{sgn}\left(\xi_{1}\right)\right] \\
& =s_{1}^{T} g_{1}\left[\zeta_{2} \rho_{2}-k_{1} s_{1}-\tau_{1} \operatorname{sig}^{\gamma}\left(s_{1}\right)-\zeta_{1} \dot{\rho}_{1}+l_{1} \operatorname{sgn}\left(\xi_{1}\right)\right] \\
& =s_{1}^{T} g_{1}\left[F_{1}-k_{1} s_{1}-\tau_{1} \operatorname{sig}^{\gamma}\left(s_{1}\right)\right]
\end{aligned}
$$

where $F_{1}=\left[F_{11}, F_{12}, F_{13}\right]^{T}=\zeta_{2} \rho_{2}-\zeta_{1} \dot{\rho}_{1}+l_{1} \operatorname{sgn}\left(\xi_{1}\right)$, $\zeta_{1 i}$ and $\zeta_{2 i}$ are bounded by $\left|\zeta_{1 i}\right|<\min \{\underline{\delta}, \bar{\delta}\}$ and $\left|\zeta_{2 i}\right|<$ $\min \{\underline{\delta}, \bar{\delta}\}$. Since $\rho_{1}$ and $\rho_{2}$ are decreasing functions, which further implied that $\dot{\rho}_{1}$ and $\dot{\rho}_{2}$ are also bounded. Besides, $l_{1} \operatorname{sgn}\left(\xi_{1 i}\right) \leq l_{1}$, and $l_{1}$ is design parameter. According to the Extreme Value Theorem [29], there is positive constant $\bar{F}_{1}>0$ satisfying the following inequality for $\forall t \in\left[0, \tau_{\max }\right)$

$\left|F_{1 i}\right|=\left|\zeta_{2 i} \rho_{2}-\zeta_{1 i} \dot{\rho}_{1}+l_{1} \operatorname{sgn}\left(\xi_{1 i}\right)\right| \leq \bar{F}_{1}$

Substituting (56) into (54) yields

$\dot{V}_{2} \leq \sum_{i=1}^{3} g_{1 i}\left(\bar{F}_{1}\left|s_{1 i}\right|-k_{1} s_{1 i}^{2}-\tau_{1}\left|s_{1 i}\right|^{1+\gamma}\right)$

From (57), it can be deduced that $\dot{V}_{2} \leq \sum_{i=1}^{3} g_{1 i}\left(\bar{F}_{1}\left|s_{1 i}\right|-\right.$ $k_{1} s_{1 i}^{2}$ ), which implies that $\dot{V}_{2}$ is negative for the case $s_{1 i}>\frac{\bar{F}_{1}}{k_{1}}$. Consequently, it can be concluded that $s_{1 i}$ will converge to a compact set $\Omega_{1}=\left\{s_{1 i}|| s_{1 i} \mid \leq s_{M 1}\right\}$ with $s_{M 1}=\max \left\{\left|s_{1 i}(0)\right|, \frac{\bar{F}_{1}}{k_{1}}\right\}$ for $\forall t \in\left[0, \tau_{\max }\right)$. According to Theorem 1 and (34), one can obtain that $\varepsilon_{1 i}$ is bounded by $\left|\varepsilon_{1 i}\right| \leq \varepsilon_{M 1}$, and $\varepsilon_{M 1}>0$ is a positive constant. Moreover, it is concluded from (33) that the virtual control $\alpha$ is bounded for $\forall t \in\left[0, \tau_{\max }\right)$.

From (28), taking the inverse tangent function yields

$-\underline{\delta}<\frac{e^{-2 \varepsilon_{M 1} \bar{\delta}}-\underline{\delta}}{e^{-2 \varepsilon_{M 1}}+1}=\zeta_{m 1} \leq \zeta_{1 i}(t) \leq \zeta_{M 1}=\frac{e^{2 \varepsilon_{M 1} \bar{\delta}}-\underline{\delta}}{e^{2 \varepsilon_{M 1}}+1} \leq \bar{\delta}-\underline{\delta}<\frac{e^{-2 \varepsilon_{M 2} \bar{\delta}}-\underline{\delta}}{e^{-2 \varepsilon_{M 2}}+1}=\zeta_{m 2} \leq \zeta_{2 i}(t) \leq \zeta_{M 2}=\frac{e^{2 \varepsilon_{M 2}} \bar{\delta}-\underline{\delta}}{e^{2 \varepsilon_{M 2}}+1} \leq \bar{\delta}$

for $\forall t \in\left[0, \tau_{\max }\right)$. According to the definition $\zeta_{1 i}=\frac{z_{1 i}}{\rho_{1}}$, it is obtained that $-\underline{\delta} \rho_{1}(t)<e_{i}(t)<\bar{\delta} \rho_{1}(t), i=1,2,3$ for $\forall t \in\left[0, \tau_{\max }\right)$, which implies that attitude tracking error $e_{i}$ can be retained within the predefined boundary for $\forall t \in\left[0, \tau_{\max }\right)$.

$V_{3}=\frac{1}{2} \varepsilon_{2}^{T} \varepsilon_{2}$

Differentiating (59) yields

$\dot{V}_{3}=\varepsilon_{2}^{T} \dot{\varepsilon}_{2}$

Substituting (44) into (60) yields

$$
\begin{aligned}
\dot{V}_{3}= & \varepsilon_{2}^{T} g_{2}\left[\Phi\left(x_{1}, x_{2}, \omega_{d}, \dot{\omega}_{d}\right)+G\left(x_{1}\right) J^{-1} d-k_{2} \varepsilon_{2}-\right. \\
& \left.\tau_{2} \operatorname{sig}^{\gamma}\left(\varepsilon_{2}\right)-\dot{x}_{c}-\zeta_{2} \dot{\rho}_{2}\right] \\
= & \varepsilon_{2}^{T} g_{2} \cdot\left[F_{2}-k_{2} \varepsilon_{2}-\tau_{2} \operatorname{sig}^{\gamma}\left(\varepsilon_{2}\right)\right]
\end{aligned}
$$

where $F_{2}=\left[F_{21}, F_{22}, F_{23}\right]^{T}=\Phi\left(x_{1}, x_{2}, \omega_{d}, \dot{\omega}_{d}\right)-\dot{x}_{c}-$ $\zeta_{2} \dot{\rho}_{2}+G\left(x_{1}\right) J^{-1} d$ is the lumped unknown dynamics. Following the similar analysis given in Step 1 and using Lemma 1 and Lemma 2, it is obtained that the signals $\zeta_{2 i}, \rho_{2}, \dot{\rho}_{2}, x_{c i}$ and $\dot{x}_{c i}$ are bounded for $\forall t \in\left[0, \tau_{\max }\right)$. Moreover, the external disturbance $d$ is bounded, and $\Phi\left(x_{1}, x_{2}, \omega_{d}, \dot{\omega}_{d}\right)$ is a continuous function of $x_{1}, x_{2}, \omega_{d}$ and $\dot{\omega}_{d}$, which is bounded for $\forall t \in\left[0, \tau_{\max }\right)$. Then, from the Extreme Value Theorem in [29], there is a positive constant $\bar{F}_{2}>0$, such that the following inequality holds for $\forall t \in\left[0, \tau_{\max }\right)$

$\left|F_{2 i}\right|=\left|\Phi_{i}-\dot{x}_{c i}-\zeta_{2 i} \dot{\rho}_{2}\right| \leq \bar{F}_{2}$

Substituting (62) into (61) yields

$\dot{V}_{3} \leq \sum_{i=1}^{3} g_{2 i}\left(\bar{F}_{2}\left|\varepsilon_{2 i}\right|-k_{2} \varepsilon_{2 i}^{2}-\tau_{2}\left|\varepsilon_{2 i}\right|^{1+\gamma}\right)$

From (63), it can be deduced that $\dot{V}_{3} \leq \sum_{i=1}^{3} g_{2 i}\left(\bar{F}_{2}\left|\varepsilon_{2 i}\right|\right.$ $-k_{2} \varepsilon_{2 i}^{2}$ ), which implies that $\dot{V}_{2}$ is negative for the case $s_{2 i}>\frac{F_{2}}{k_{2}}$. Consequently, it can be concluded that $\varepsilon_{2 i}$ will converge to a compact set $\Omega_{2}=\left\{\varepsilon_{2 i}|| \varepsilon_{2 i} \mid \leq \varepsilon_{M 2}\right\}$ with $\varepsilon_{M 2}=\max \left\{\left|\varepsilon_{2 i}(0)\right|, \frac{\bar{F}_{2}}{k_{2}}\right\}$ for $\forall t \in\left[0, \tau_{\max }\right)$. Thus, $\varepsilon_{2 i}$ is bounded, and the actual control law $u$ in (43) is also bounded for $\forall t \in\left[0, \tau_{\max }\right)$.

From (39), taking the inverse tangent function yields
Step 2: Selecting the Lyapunov function as for $\forall t \in\left[0, \tau_{\max }\right)$. According to the definition $\zeta_{2 i}=\frac{z_{2 i}}{\rho_{2}}$, one can obtain that $-\underline{\delta} \rho_{2}(t)<z_{2 i}(t)<\bar{\delta} \rho_{2}(t), i=$ 
$1,2,3$, which implies that $x_{2 i}-x_{c i}, i=1,2,3$ can be retained within the predefined boundary for $\forall t \in\left[0, \tau_{\max }\right)$.

Step 3: In this step, we will show that $\tau_{\max }=$ $+\infty$. From (58) and (64), it is obtained that $\zeta(t) \in$ $\bar{\Omega}_{\zeta}, \forall \in\left[0, \tau_{\max }\right)$, where the set $\bar{\Omega}_{\zeta}=\left[\zeta_{m 1}, \zeta_{M 1}\right]_{1} \times$ $\ldots \times\left[\zeta_{m 2}, \zeta_{M 2}\right]_{6}$ is nonempty and compact. According to the definition of $\Omega_{\zeta}$, it is clear that $\bar{\Omega}_{\zeta} \subset \Omega_{\zeta}$ holds. Thus, if we assume $\tau_{\max }<\infty$ and consider the fact $\bar{\Omega}_{\zeta} \subset \Omega_{\zeta}$, Proposition 1 indicates that there exists a time $t_{1} \in\left[0, \tau_{\max }\right)$ such that $\zeta\left(t_{1}\right) \notin \bar{\Omega}_{\zeta}$, which creates an obvious contradiction. Therefore, the opposite of the assumption is true, i.e., $\tau_{\max }=\infty$ holds. Then, it can be concluded that all signals in the closed-loop systems are bounded, and the attitude tracking error $e_{i}$ is maintained within the predefined boundary for $\forall t \geq 0$.

Step 4: Construct the following Lyapunov function

$V_{4}=V_{2}+V_{3}$

From (57) and (63), the derivative of $V_{4}$ is obtained as

$$
\begin{aligned}
\dot{V}_{4}= & \dot{V}_{2}+\dot{V}_{3} \\
\leq & \sum_{i=1}^{3} g_{1 i}\left(\bar{F}_{1}\left|s_{1 i}\right|-k_{1} s_{1 i}^{2}-\tau_{1}\left|s_{1 i}\right|^{1+\gamma}\right)+ \\
& \sum_{i=1}^{3} g_{2 i}\left(\bar{F}_{2}\left|\varepsilon_{2 i}\right|-k_{2} \varepsilon_{2 i}^{2}-\tau_{2}\left|\varepsilon_{2 i}\right|^{1+\gamma}\right)
\end{aligned}
$$

Introducing two auxiliary terms $\frac{\bar{F}_{1}^{2}}{4 k_{12}^{2}}$ and $\frac{\bar{F}_{2}^{2}}{4 k_{22}^{2}}$ into (66) leads to

$$
\begin{aligned}
\dot{V}_{4}= & \sum_{i=1}^{3} g_{1 i}\left[-k_{11} s_{1 i}^{2}-k_{12}\left(\left|s_{1 i}\right|-\frac{\bar{F}_{1}}{2 k_{12}}\right)^{2}+\frac{\bar{F}_{1}^{2}}{4 k_{12}^{2}}-\right. \\
& \left.\tau_{1}\left|s_{1 i}\right|^{1+\gamma}\right]+\sum_{i=1}^{3} g_{2 i}\left[-k_{21} \varepsilon_{2 i}^{2}-k_{22}\left(\left|\varepsilon_{2 i}\right|-\frac{\bar{F}_{2}}{2 k_{22}}\right)^{2}\right. \\
& \left.+\frac{\bar{F}_{2}^{2}}{4 k_{22}^{2}}-\tau_{2}\left|\varepsilon_{2 i}\right|^{1+\gamma}\right] \\
\leq & \sum_{i=1}^{3} g_{1 i}\left(-k_{11} s_{1 i}^{2}-\tau_{1}\left|s_{1 i}\right|^{1+\gamma}+\frac{\bar{F}_{1}^{2}}{4 k_{12}}\right)+ \\
& \sum_{i=1}^{3} g_{2 i}\left(-k_{21} \varepsilon_{2 i}^{2}-\tau_{2}\left|\varepsilon_{2 i}\right|^{1+\gamma}+\frac{\bar{F}_{2}^{2}}{4 k_{22}}\right) \\
\leq & \mu_{1} V_{4}-\mu_{2} V_{4}^{\frac{1+\gamma}{2}}+\varphi
\end{aligned}
$$

where $k_{1}=k_{11}+k_{12}, k_{2}=k_{21}+k_{22}, \mu_{1}=\min \left\{2 g_{m 1} k_{11}\right.$, $\left.2 g_{m 2} k_{21}\right\}, \mu_{2}=2^{\frac{1+\gamma}{2}} \cdot \min \left\{\tau_{1} g_{m 1}, \tau_{2} g_{m 2}\right\}$, and $\varphi=$ $\frac{3 g_{M 1} \bar{M}_{1}^{2}}{4 k_{12}}+\frac{3 g_{M 2} \bar{M}_{2}^{2}}{4 k_{22}}$. From Lemma 3 , it follows that $V_{4}$ will converge to the region $V_{4} \leq \min \left\{\frac{\varphi}{(1-\theta) \mu_{1}},\left(\frac{\varphi}{(1-\theta) \mu_{2}}\right)^{\frac{2}{\gamma+1}}\right\}$ in finite time, where $0<\theta<1$. It means that $s_{1 i}$ and $\varepsilon_{2 i}$ will converge to a sufficiently small region around zero within the finite time $T_{2}$ given by

$$
\begin{aligned}
T_{2} \leq & \max \left\{\frac{2}{\mu_{1} \theta(1-\gamma)} \ln \left(1+\frac{\theta \mu_{1}}{\mu_{2}}\left[V_{4}(0)\right]^{\frac{1-\gamma}{2}}\right),\right. \\
& \left.\frac{2}{\mu_{1}(1-\gamma)} \ln \left(1+\frac{\mu_{1}}{\theta \mu_{2}}\left[V_{4}(0)\right]^{\frac{1-\gamma}{2}}\right)\right\}
\end{aligned}
$$

From (67), it is obtained that $s_{1 i}$ and $\varepsilon_{2 i}$ can both converge into a small region within a finite time. According to (34) and (51), the constrained errors $\varepsilon_{1 i}$ can also converge into a sufficiently small region around zero within a finite time. According to the definition $x_{2}=z_{2}+x_{c}$ and $\omega_{e}=G\left(x_{1}\right)^{-1} x_{2}$, it can be obtained that $x_{2}$ is bounded, which further implies that angular velocity error $\omega_{e}$ is bounded. Consequently, the attitude tracking error $e_{i}$ is maintained within the prescribed region (22) and converge into the sufficiently small region around origin in finite time $T_{3} \leq T_{1}+T_{2}$. This completes the proof.

Remark 6: The upper bound of the error convergence time is determined by the choice of some control parameters, such as $k_{1}, k_{2}, \tau_{1}$ and $\tau_{2}$, which can be specified by the users in prior. Generally, in order to guarantee the attitude tracking error $e_{i}, i=1,2,3$ converge into a sufficiently small region, larger values of parameters $k_{1}, k_{2}, \tau_{1}$ and $\tau_{2}$ are suggested in (33) and (43), respectively. However, too large $k_{1}, k_{2}, \tau_{1}$ and $\tau_{2}$ may lead to a high control gain in the starting phase. Consequently, a tradeoff between the convergence time and control gain should be made for the selections of the parameters $k_{1}, k_{2}, \tau_{1}$ and $\tau_{2}$.

\section{Simulation Results}

In this section, two different control schemes are provided, including the proposed FTAFC (M1) in this paper, and standard AFC (M2) in [31]. Detailed control configurations and corresponding discussions are given as follows.

\subsection{Different control schemes}

1) The proposed M1 scheme: The proposed M1 scheme consists of the virtual control law (33) and actual control signal (43), of which the parameters are set as $k_{1}=0.15, k_{2}=0.5, \tau_{1}=0.1, \tau_{2}=0.1$ and $\gamma=\frac{3}{5}$. The parameters of $\rho_{1}(t)$ and $\rho_{2}(t)$ defined in (19) are chosen as $T_{01}=T_{02}=5, \rho_{T_{01}}=0.005, \rho_{T_{02}}=0.01, \rho_{01}=$ $0.6, \rho_{02}=0.4, \underline{\delta}=\bar{\delta}=1$ and $c=1$. Moreover, the parameters of finite-time command filter (32) and error compensation signal (35) are selected as $\beta_{1}=3, \beta_{2}=4$ and $l_{1}=0.1$. 


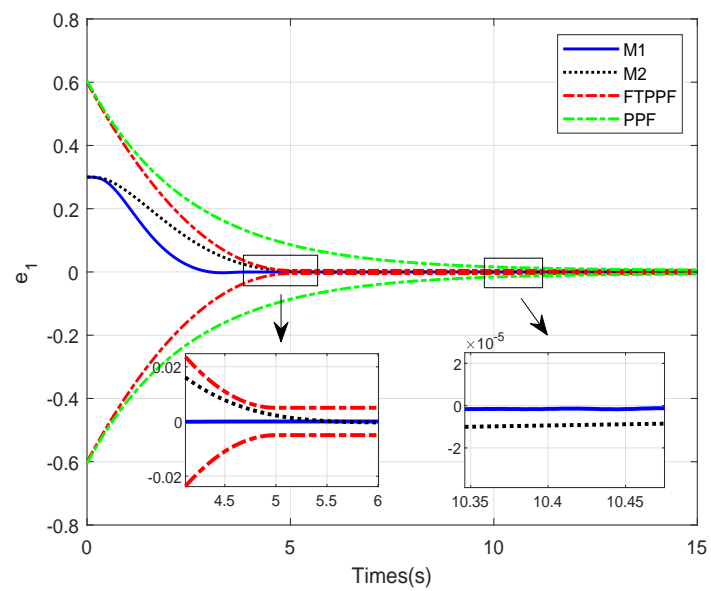

Fig. 2 Comparison of the attitude tracking error $e_{1}$

3) The M2 scheme: In the M2 scheme [31], the virtual controller and actual controller are designed as $\alpha=$ $-k_{1} \varepsilon_{1}$ and $u=-k_{2} J \varepsilon_{2}$, respectively. For fair comparison, the parameters used in both M1 and M2 schemes are set the same, for example, i.e., the control gains $k_{1}=0.15, k_{2}=0.5$. The parameters of $\rho_{1}(t)$ and $\rho_{2}(t)$ defined in $(21)$ are chosen as $a_{1}=0.4, a_{2}=0.5, \rho_{10}=$ $0.6, \rho_{20}=0.4, \rho_{1 \infty}=0.005, \rho_{2 \infty}=0.01, \underline{\delta}=\bar{\delta}=1$.

For the spacecraft system (11), the initial values of attitude and angular velocity are given as $\sigma(0)=$ $[0.3,-0.2,-0.3]^{T}$ and $\omega(0)=[0,0,0]^{T} \mathrm{rad} / \mathrm{s}$ respectively, and the desired angular velocity is selected as

$$
\omega_{d}=0.05 *\left[\begin{array}{l}
0.2 \sin \left(\frac{\pi t}{100}\right) \\
0.3 \sin \left(\frac{2 \pi t}{100}\right) \\
0.5 \sin \left(\frac{3 \pi t}{100}\right)
\end{array}\right] \mathrm{rad} / \mathrm{s} .
$$

and the inertia matrix $J$ and the external disturbance $d$ are chosen as

$$
J=\left[\begin{array}{ccc}
20 & 1.2 & 0.9 \\
1.2 & 17 & 1.4 \\
0.9 & 1.4 & 15
\end{array}\right] \mathrm{kg} \cdot \mathrm{m}^{2}
$$

and

$$
d=\left[\begin{array}{c}
0.2 \sin (0.1 t) \\
0.3 \sin (0.2 t) \\
0.5 \sin (0.2 t)
\end{array}\right] \mathrm{N} \cdot \mathrm{m}
$$

respectively.

The corresponding simulation results are depicted by Figs.2-9. Fig.2 shows the comparison of the attitude tracking error $e_{1}$. From Fig.2, it is seen that the proposed M1 scheme can guarantee a better tracking performance than M2, that is a faster tracking speed and smaller tracking errors can be achieved with M1 scheme. Moreover, M1 and M2 strictly retain the attitude tracking errors within the region (22) specified by the FTPPF and PPF, respectively. However, the

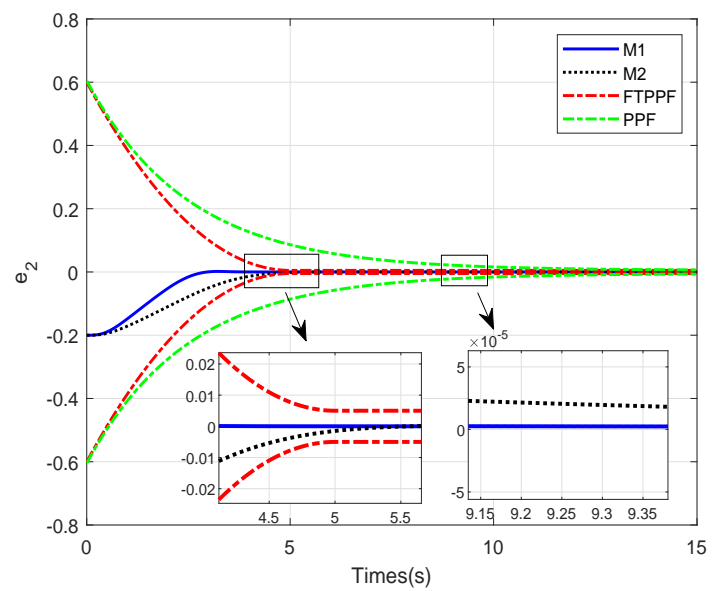

Fig. 3 Comparison of the attitude tracking error $e_{2}$

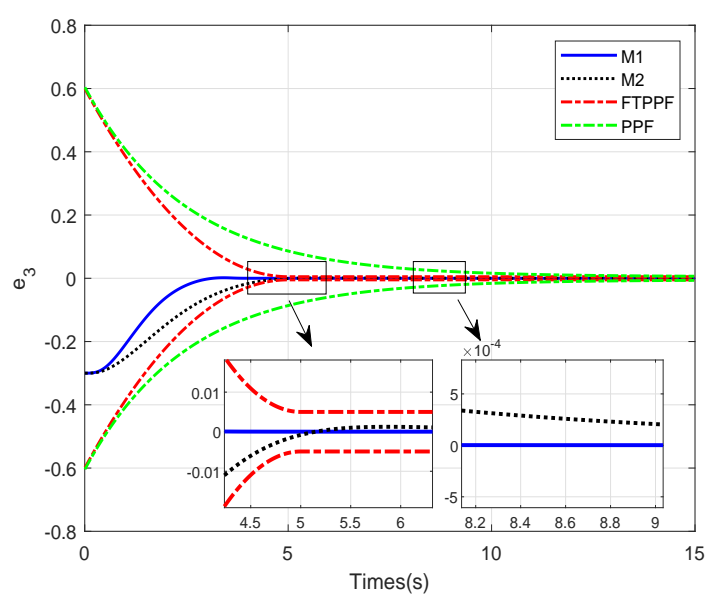

Fig. 4 Comparison of the attitude tracking error $e_{3}$

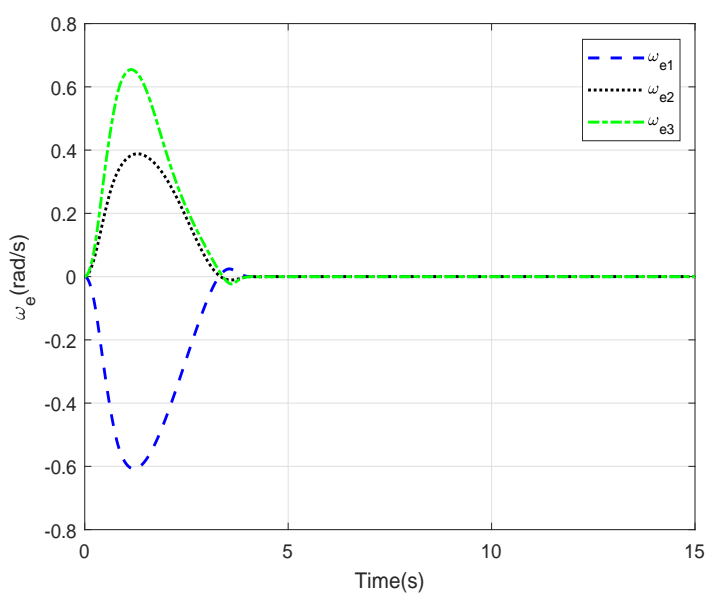

Fig. 5 Angular velocity error $\omega_{e}$ of the M1 scheme

settling time of FTPPF in M1 is fixed, while the settling time of PPF in M2 is infinite, and the improved transient convergence over the standard AFC can be 


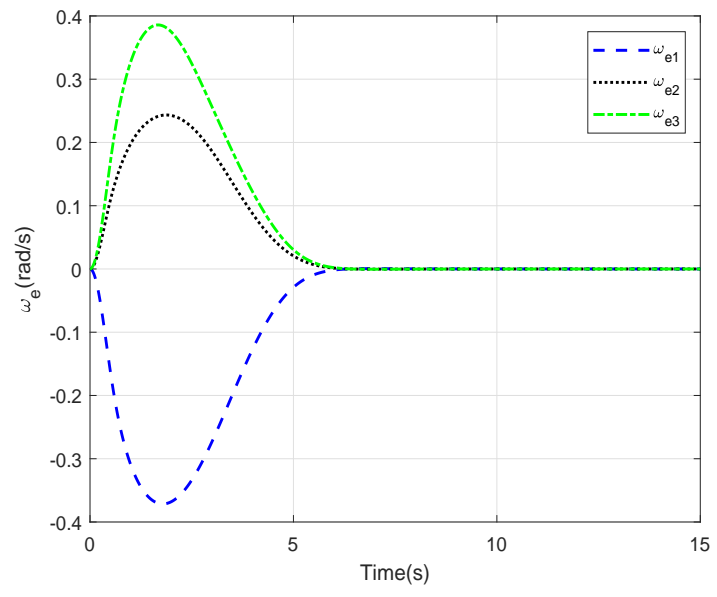

Fig. 6 Angular velocity error $\omega_{e}$ of the M2 scheme

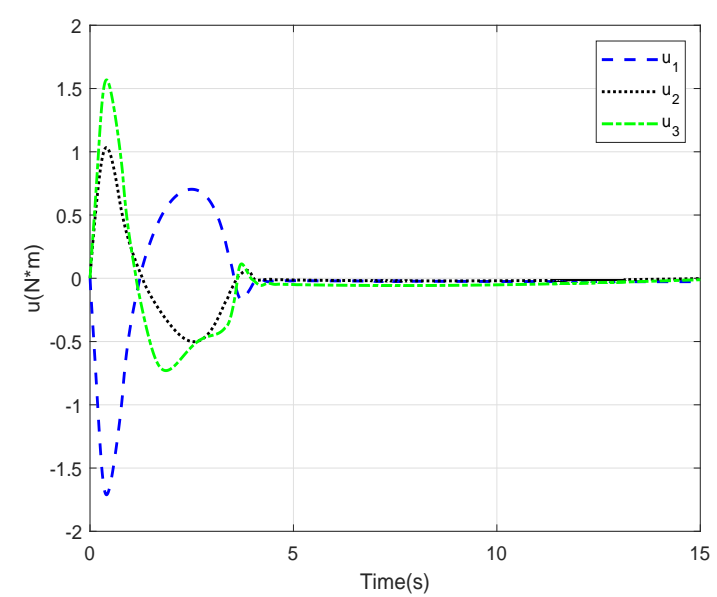

Fig. 7 Control input $u$ of the M1 scheme

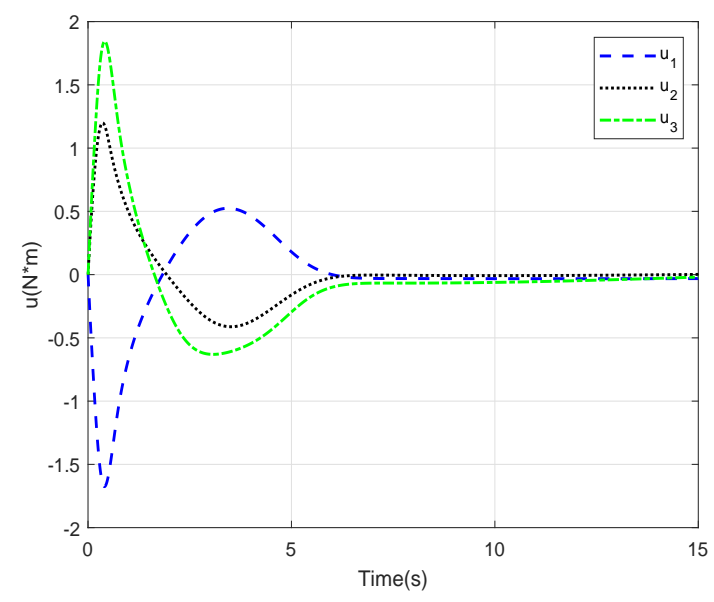

Fig. 8 Control input $u$ of the M2 scheme in the FTAFC. Similar results can be found in Fig.3, which describes the comparison of the attitude tracking

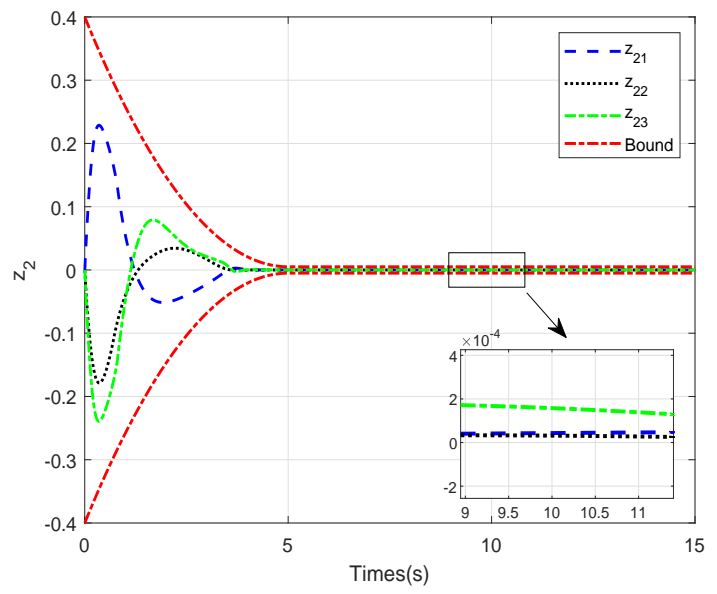

Fig. 9 Intermediate error $z_{2}$ of the M1 scheme

error $e_{2}$. The comparison of the attitude tracking error $e_{3}$ is shown in Fig.4, and again one can find that the proposed M1 scheme can guarantee a better tracking performance with relatively smaller steady-state tracking error than M2 scheme. The angular velocity error $\omega_{e}$ of M1 scheme and M2 scheme are shown in Fig.5 and Fig.6, respectively. It is seen from Fig.5 and Fig.6 that the settling time of the proposed M1 scheme is shorter than M2 scheme. Fig.7 and Fig.8 show that the control torques of the compared two schemes are almost the same. Fig.9 depicts the intermediate error $z_{2}$ of the proposed M1 scheme. We can find from Fig.9 that the intermediate error $z_{2}$ can converge into the predefined region within a finite time.

From Figs.2-9, it can be concluded that by employing the FTPPF to constrain the attitude tracking errors, M1 can achieve smaller attitude tracking errors than M2. Moreover, the attitude tracking speed of the proposed M1 is faster than that of M2 with the help of the finite-time control technique. It means that a better steady-state attitude tracking performance is achieved with the proposed M1 scheme in this paper.

\section{Conclusion}

This paper has proposed a finite-time command-filtered approximation-free attitude tracking control scheme for rigid spacecraft without using any function approximations. A novel finite-time PPF is first presented to ensure that the attitude tracking errors converge into the predefined region within finite time. To overcome the singularity caused by differentiating the virtual control signals, a finite-time error compensation mechanism is developed and incorporated into the recursive control design. However, the state values of attitude and angu- 
lar velocity should be available to design the finite-time controller which may limit its practical applications. Consequently, the finite-time approximation-free attitude tracking control without velocity measurements is still a challenging work, and deserves to be further investigated in future work.

\section{Acknowledgment}

The authors would thank the support from the National Natural Science Foundation (NNSF) of China under Grant Nos. 61973274 and 61873239, the Key Laboratory Open Project Fund under Grant No. GDSC202010, and the Anhui Provincial Natural Science Foundation under Grant No.2008085QF331.

\section{Conflict of interest}

The authors declare that they have no conflict of interest.

\section{Data Availability Statements}

The datasets generated during and/or analysed during the current study are not publicly available as the data also forms part of an ongoing study, but are available from the corresponding author on reasonable request.

\section{References}

1. P. C. Hughes, Spacecraft attitude dynamics, New York: Wiley, 1986.

2. Z. Chen and J. Huang, "Attitude tracking and disturbance rejection of rigid spacecraft by adaptive control," IEEE Transactions on Automatic Control, vol. 54, no. 3, pp. 600-605, March 2009.

3. Q. Hu and B. Xiao, "Fault-tolerant sliding mode attitude control for flexible spacecraft under loss of actuator effectiveness," Nonlinear Dynamics, vol. 64, no. 1, pp. 13-23, 2011.

4. Q. Shen, D. Wang, S. Zhu, and E. K. Poh, "Robust control allocation for spacecraft attitude tracking under actuator faults," IEEE Transactions on Control Systems Technology, vol. 25, no. 3, pp. 1068-1075, 2016.

5. C. Wang, L. Guo, C. Wen, Q. Hu, and J. Qiao, "Eventtriggered adaptive attitude tracking control for spacecraft with unknown actuator faults," IEEE Transactions on Industrial Electronics, vol. 67, no. 3, pp. 2241-2250, March 2020.

6. S. Wu and S. Wen, "Robust $H_{\infty}$ output feedback control for attitude stabilization of a flexible spacecraft," Nonlinear Dynamics, vol. 84, no. 1, pp. 405-412, 2016.

7. A.-M. Zou, K. D. Kumar, and A. H. de Ruiter, "Finitetime spacecraft attitude control under input magnitude and rate saturation," Nonlinear Dynamics, vol. 99, no. 3, pp. 2201-2217, 2020.
8. Q. Hu and X. Shao, "Smooth finite-time fault-tolerant attitude tracking control for rigid spacecraft," Aerospace Science and Technology, vol. 55, pp. 144-157, August 2016.

9. M. Sun, "Two-phase attractors for finite-duration consensus of multiagent systems," IEEE Transactions on Systems Man and Cybernetics: Systems, vol. 50, no. 5, pp. 1757-1765, May 2020.

10. K. Lu and Y. Xia, "Adaptive attitude tracking control for rigid spacecraft with finite-time convergence," Automatica, vol. 49, no. 12, pp. 3591-3599, December 2013.

11. L. Zhao, J. Yu, and H. Yu, "Adaptive finite-time attitude tracking control for spacecraft with disturbances," IEEE Transactions on Aerospace and Electronic Systems, vol. 54, no. 3, pp. 1297-1305, June 2018.

12. J. Zhang, W. Zhao, G. Shen, and Y. Xia, "Disturbance observer-based adaptive finite-time attitude tracking control for rigid spacecraft," IEEE Transactions on Systems, Man, and Cybernetics, to be published, doi: 10.1109/TSMC.2019.2947320, 2020.

13. Q. Chen, H. Shi, and M. Sun, "Echo state networkbased backstepping adaptive iterative learning control for strict-feedback systems: An error-tracking approach," IEEE Transactions on Cybernetics, vol. 50, no. 7, pp. 3009-3022, July 2020.

14. S. Wang, J. Na, and X. Ren, "Rise-based asymptotic prescribed performance tracking control of nonlinear servo mechanisms," IEEE Transactions on Systems, Man, and Cybernetics: Systems, vol. 48, no. 12, pp. 2359-2370, December 2018.

15. J. Na, S. Wang, Y. J. Liu, Y. Huang, and X. Ren, "Finitetime convergence adaptive neural network control for nonlinear servo systems," IEEE Transactions on Cybernetics, vol. 50, no. 6, pp. 2568-2579, June 2020.

16. X. Zhou, C. Gao, Z. Li, X. Ouyang, and L. Wu, "Observer-based adaptive fuzzy finite-time prescribed performance tracking control for strict-feedback systems with input dead-zone and saturation," Nonlinear Dynamics, vol. 103, no. 2, pp. 1645-1661, 2021.

17. S. Xie and Q. Chen, "Adaptive nonsingular predefinedtime control for attitude stabilization of rigid spacecrafts," IEEE Transactions on Circuits and Systems II: Express Briefs, to be published, doi: 10.1109/TCSII.2021.3078708, 2021.

18. Q. Chen, M. Tao, X. He, and L. Tao, "Fuzzy adaptive nonsingular fixed-time attitude tracking control of quadrotor uavs," IEEE Transactions on Aerospace and Electronic systems, to be published, doi: 10.1109/TAES.2021.3067610, 2021.

19. A. M. Zou, K. D. Kumar, Z. G. Hou, and X. Liu, "Finitetime attitude tracking control for spacecraft using terminal sliding mode and chebyshev neural network," IEEE Transactions on Systems, Man, And Cybernetics, vol. 41, no. 4, pp. 950-63, August 2011.

20. B. Huo, Y. Xia, K. Lu, and M. Fu, "Adaptive fuzzy finitetime fault-tolerant attitude control of rigid spacecraft," Journal of the Franklin Institute, vol. 352, no. 10, pp. 4225-4246, October 2015.

21. C. P. Bechlioulis and G. A. Rovithakis, "Robust adaptive control of feedback linearizable mimo nonlinear systems with prescribed performance," IEEE Transactions on Automatic Control, vol. 53, no. 9, pp. 2090-2099, October 2008 .

22. M. Chen, X. Liu, and H. Wang, "Adaptive robust faulttolerant control for nonlinear systems with prescribed performance," Nonlinear Dynamics, vol. 81, no. 4, pp. 1727-1739, 2015. 
23. S. Wang, J. Na, and Q. Chen, "Adaptive predefined performance sliding mode control of motor driving systems with disturbances," IEEE Transactions on Energy Conversion, to be published, doi:10.1109/TEC.2020.3038010, 2020.

24. L. Zhang and G.-H. Yang, "Adaptive fuzzy prescribed performance control of nonlinear systems with hysteretic actuator nonlinearity and faults," IEEE Transactions on Systems, Man, and Cybernetics: Systems, vol. 48, no. 12, pp. 2349-2358, December 2018.

25. C. Zhang, G. Ma, Y. Sun, and C. Li, "Prescribed performance adaptive attitude tracking control for flexible spacecraft with active vibration suppression," Nonlinear Dynamics, vol. 96, no. 3, pp. 1909-1926, 2019.

26. Y.-J. Liu, Q. Zeng, S. Tong, C. L. P. Chen, and L. Li$\mathrm{u}$, "Actuator failure compensation-based adaptive control of active suspension systems with prescribed performance," IEEE Transactions on Industrial Electronics, vol. 67, no. 8, pp. 7044-7053, August 2020.

27. S. Sui, C. P. Chen, and S. Tong, "A novel adaptive nn prescribed performance control for stochastic nonlinear systems," IEEE Transactions on Neural Networks and Learning Systems, to be published, doi: 10.1109/TNNLS.2020.3010333, 2020.

28. Y. Liu, X. Liu, Y. Jing, and Z. Zhang, "A novel finitetime adaptive fuzzy tracking control scheme for nonstrict feedback systems," IEEE Transactions on Fuzzy Systems, vol. 27, no. 4, pp. 646-658, April 2019.

29. C. P. Bechlioulis and G. A. Rovithakis, "A lowcomplexity global approximation-free control scheme with prescribed performance for unknown pure feedback systems," Automatica, vol. 50, no. 4, pp. 1217-1226, April 2014.

30. Y. H. Choi and S. J. Yoo, "Decentralized approximationfree control for uncertain large-scale pure-feedback systems with unknown time-delayed nonlinearities and control directions," Nonlinear Dynamics, vol. 85, no. 2, pp. 1053-1066, July 2016.

31. Y. Huang, J. Na, X. Wu, and G. Gao, "Approximationfree control for vehicle active suspensions with hydraulic actuator," IEEE Transactions on Industrial Electronics, vol. 65 , no. 9, pp. 7258-7267, September 2018.

32. C. Zhang, J. Na, J. Wu, Q. Chen, and Y. Huang, "Proportional-integral approximation-free control of robotic systems with unknown dynamics," IEEE/ASME Transactions on Mechatronics, to be published, doi: 10.1109/TMECH.2020.3035660, 2020.

33. S. I. Han and J. Lee, "Approximation-free tracking error constraint control for lagrangian systems," IEEE Transactions on Industrial Electronics, vol. 64, no. 2, pp. 12691278, Feburary 2017.

34. J. Luo, Z. Yin, C. Wei, and J. Yuan, "Low-complexity prescribed performance control for spacecraft attitude stabilization and tracking," Aerospace Science and Technology, vol. 74, pp. 173-183, March 2018.

35. Y. Hu, Y. Geng, B. Wu, and D. Wang, "Model-free prescribed performance control for spacecraft attitude tracking," IEEE Transactions on Control Systems Technology, vol. 29, no. 1, pp. 165-179, January 2021.

36. H. Wong, M. S. D. Queiroz, and V. Kapila, "Adaptive tracking control using synthesized velocity from attitude measurements," in American Control Conference, vol. 37, no. 6, June 2001, pp. 947-953.

37. Q. Chen, S. Xie, and X. He, "Neural-network based adaptive singularity-free fixed-time attitude tracking control for spacecrafts," IEEE Transactions on Cybernetics, to be published, doi: 10.1109/TCYB.2020.3024672, 2020.
38. J. L. Crassidis and F. L. Markley, "Sliding mode control using modified rodrigues parameters," Journal of Guidance Control and Dynamics, vol. 9, no. 6, pp. 1381-1383, November 1996.

39. M. D. Shuster, "A survey of attitude representations," $J$ of Astronautical Sciences, vol. 41, no. 4, pp. 439-517, October 1993.

40. E. D. Sontag, Mathematical Control Theory, London, U.K.: Springer, 1998.

41. Levant and Arie, "Higher-order sliding modes, differentiation and output-feedback control," International Journal of Control, vol. 76, no. 9-10, pp. 924-941, July 2003.

42. C. Yang, Y. Jiang, W. He, J. Na, Z. Li, and B. Xu, "Adaptive parameter estimation and control design for robot manipulators with finite-time convergence," IEEE Transactions on Industrial Electronics, vol. 65, no. 10, pp. 8112-8123, October 2018. 